\title{
Réhabilitation de la diversité et besoin d'unité en biologie
}

\section{Maryline Cantor}

\section{(2) OpenEdition}

1 Journals

\section{Édition électronique}

URL : http://journals.openedition.org/trema/2009

DOI : 10.4000/trema.2009

ISSN : 2107-0997

\section{Éditeur}

Faculté d'Éducation de l'université de Montpellier

\section{Édition imprimée}

Date de publication : 1 septembre 1996

Pagination : 55-64

ISSN : 1167-315X

\section{Référence électronique}

Maryline Cantor, «Réhabilitation de la diversité et besoin d'unité en biologie », Tréma [En ligne],

9-10 | 1996, mis en ligne le 11 septembre 2013, consulté le 19 avril 2019. URL : http:// journals.openedition.org/trema/2009; DOI : 10.4000/trema.2009

Ce document a été généré automatiquement le 19 avril 2019.

Trema 


\title{
Réhabilitation de la diversité et besoin d'unité en biologie
}

\author{
Maryline Cantor
}

\section{Introduction}

1 «Unité et diversité du vivant à tous les niveaux d'intégration » : ce concept apparemment paradoxal reste fondamental de la biologie moderne (F. Jacob, 1970). Tel un pendule, la pensée biologique oscille sans cesse entre l'unité du vivant dans sa substance et son fonctionnement, et la multiplicité concrète des formes et de ses manifestations. Suivant les périodes et selon les différents domaines d'études biologiques, la priorité a souvent été donnée à l'un ou l'autre aspect. Ces dernières années, l'approche « unitaire » semblait être accentuée, au détriment de la compréhension de la diversité du vivant. Cependant de nombreux biologistes évolutionnistes (E. Mayr, 1982, 1991 ; S.J. Gould, 1987, 1995), mais aussi des spécialistes de biologie moléculaire (P. Kourilsky, 1993) et de génétique (B. Macclintock ; 1929, 1951), montrent les intérêts de l'approche naturaliste. La recherche biomédicale, de son côté, s'investit dans l'appréhension de la diversité et de la variabilité, pour comprendre par exemple les maladies féminines longtemps délaissées, et la recherche militaire envisage la mise au point d'éventuelles armes biologiques spécifiques pour certaines ethnies (Courrier International, 1995).

2 «Appréhender, au delà de sa diversité, l'unité et l'organisation du monde vivant, de la biosphère à la cellule" (projet de programme de Sciences et Technologie du collège français, mars 95); "Unicité génétique des individus et polymorphisme des espèces ", "Unité et diversité du monde vivant » (programme de Terminale scientifique des lycées français, 1994): les programmes d'enseignement français prennent en compte la «revalorisation» de la diversité. Comment concilier la structuration d'une face de ce « concept Janus » sans faire obstacle à l'autre face ? Les manuels scolaires témoignent que de nombreuses difficultés persistent pour une structuration réellement fonctionnelle de ce « concept bicéphale ». 


\section{Un peu d'histoire}

\subsection{De la diversité à l'unité}

3 La diversité du vivant et des éléments minéraux, les «curiosités de la nature » constituent le premier domaine d'interrogation des savants jusqu'au XVIII ${ }^{\mathrm{e}}$ siècle. La recherche du singulier et la valorisation de la singularité, à l'origine des Cabinets d'histoire naturelle, représentent, en fait des obstacles à la recherche d'assimilations (Canguilhem, 1968). Les problèmes d'inventaire, de classement, la recherche d'un « ordre naturel », posent ensuite la question des conditions de permanence de l'unité dans la diversité (Foucault, 1966), sous une double référence de la morphologie et de la généalogie. C'est du détour par le problème de classification que peut se fonder un domaine d'investigation ne concernant que les êtres vivants. La biologie, mot introduit en 1802 simultanément par le vitaliste Treviranus en Allemagne et par le matérialiste Lamarck en France, se présente comme une théorie unifiée des êtres vivants. Cette double introduction témoigne (Gayon, 1995) que, dès son origine, l'unité fondamentale des processus physiologiques de la vie repose sur deux hypothèses :

- une unité généalogique,

- une unité physiologique.

\subsection{Recherche de lois unitaires}

4 Tout au long du XIX ${ }^{\mathrm{e}}$ siècle, la biologie s'est cependant confondue avec uniquement la physiologie. Le positivisme se développant, la biologie s'est voulue science, c'est à dire explicative et à la recherche de lois. La physiologie, à la recherche d'un déterminisme, refuse la probabilité et se méfie de la statistique (Bernard, 1865). Les seules "lois» biologiques élaborées le sont pourtant en génétique et ce sont des lois probabilistes : les lois de Mendel. Les théories biologiques, avec des concepts essentiellement narratifs, constituent donc une alternative entre loi et histoire (Gayon, 1995).

\subsection{Unité et diversité}

Y a-t-il une biologie ou des sciences biologiques ? « La biologie se divise en deux branches [...] d'un côté on continue de s'occuper de l'organisme tout entier, considéré soit comme une unité intangible, soit comme l'élément d'une population ou d'une espèce; cette biologie fonctionne avec les concepts de l'histoire naturelle [...] De l'autre côté, au contraire, on cherche à réduire l'organisme à ses constituants » (Jacob, 1970). Tout au long du XIX ${ }^{\mathrm{e}}$ siècle la biologie s'est morcelée en de multiples sciences. Au début du XX siècle, la biochimie et la génétique, représentant chacune une des tendances de la biologie, ont pris leur essor. Actuellement, la majorité des recherches ne portent plus sur les organismes mais sur un «nouvel objet biologique » : l'ADN. « La vie est étudiée au plus près de la non-vie, à l'état maximum de dénuement de ses attributs traditionnels » (Canguilhem, 1981). Beaucoup réagissent contre ce qui peut apparaître comme une « toute puissance » de la biologie moléculaire, « cette singulière perversion de la biologie qui consiste à lui donner pour fin la négation de son objet et, par conséquent, d'ellemême en tant que science autonome » (Pichot, 1993). L'unité représente un paradigme essentiel dans la physiologie et la biologie moléculaire. Dans ces domaines prédomine la 
recherche d'un déterminisme et les investigations s'appuient essentiellement sur l'analyse et l'expérimentation.

6 Par ailleurs, la systématique, la paléontologie et l'écologie tentent de comprendre la spécificité et la variabilité, elles font souvent appel au classement et à la statistique. L'approche naturaliste se caractérise par un «principe de narrativité» qui peut apparaitre comme moins scientifique face à la recherche de «loi déterministe». C'est pourtant en raisonnant en naturaliste, en voulant comprendre l'histoire singulière de chaque grain de maïs, que Barbara Macclintock a mis en évidence les éléments transposables dans la régulation des génomes.

7 Pour Gould (1987), « la biologie forme un tout, il faut marier styles distincts de l'histoire naturelle et des expériences menées par les réductionnistes ». Mayr (1982), de son côté, pense que la "biologie n'est pas homogène ", mais par ailleurs «biologie évolutionniste et biologie fonctionnaliste ne s'excluent pas» et «en dépit d'une apparence d'émiettement continu, il y a à présent en biologie une unité bien plus grande que dans les siècles précédents $\%$.

\section{Du côté des manuels scolaires}

8 L'analyse des nouveaux manuels de lycées (éditions Bordas, Hachette et Nathan) met en évidence la persistance de plusieurs difficultés :

- la juxtaposition des aspects unité et diversité du vivant, sans essai de mise en relation,

- la survalorisation de l'unité du vivant, avec des effets de " gommage » au niveau de la présentation ou de l'explication de phénomènes biologiques (sous prétexte de simplification?)

\subsection{La partie congrue de la systématique}

Seule la collection Nathan publie un index des noms d'espèces animale ou végétale. Comprenant 71 références en classe de seconde et 19 en classe de première $S$ cet index disparaît dans le manuel de terminale $S$ ! De plus les noms d'espèces ne présentent pas toute la rigueur scientifique de la systématique (sont mélangés des noms de genres, de familles et d'ordre...). Il paraît bien difficile de structurer le concept fondamental d'espèce.

\subsection{Simplification ou dogmatisation?}

10 Sous prétexte de simplification (?), la présentation des théories et des concepts biologiques peuvent développer des conceptions fausses. Ainsi, la plupart des expériences de physiologie animale sont présentées tronquées, sans référence à des variations éventuelles et sans précisions sur le «modèle d'étude" (par exemple, les résultats concernent-ils l'individu ou l'espèce ?). Ailleurs, les représentations iconiques de la diversité des vivants au cours de l'évolution peuvent laisser supposer que la diversité du vivant concerne surtout l'époque actuelle (Voir aussi Gould, 1995).

11 Est-ce par horreur du buissonnant que les observations sur Homo sapiens neanderthalensis sont soit présentées déformées soit parfois même oubliées? 


\subsection{La statistique bafouée}

La statistique, science du particulier et de l'incertain, est sous représentée dans l'ensemble des ouvrages. D'autre part, les quelques données avancées induisent pour la plupart une non-compréhension de la pensée statistique. Alors que la moyenne et la variance représentent des doublets incontournables (Schwartz, 1994), le plus souvent seule une moyenne est présentée, avec des possibilités de confusion entre moyenne et norme.

\subsection{La génétique en question}

génétique représente une part très importante dans les nouveaux programmes de lycées. Dans tous les manuels étudiés, l'accent n'est mis que sur la stabilité du génome, aucun ne fait allusion à sa labilité et à ses interactions multiples avec l'environnement. Tous font aussi référence à l'«identité » des jumeaux. L'identité ne serait-elle donc que génétique ? Ce «mythe identitaire» oublie tous les effets de l'environnement. Ailleurs, les maladies génétiques sont expliquées comme manifestation d'une "erreur » (en référence à quelle «norme » ?).

La compréhension du caractère unique des individus fait accéder à celle de sélection naturelle. Le polymorphisme des populations, de son côté, permet de caractériser les variances élevées des phénomènes biologiques. L'exemple des populations humaines n'est pas le plus simple à étudier mais c'est une occasion de faire accéder à la pensée populationnelle : comparer la statistique des essentialistes et celle des populationnistes démontre que «les arguments fracassants de la littérature raciste se fondent presque toujours sur la pensée essentialiste » (Mayr, 1982).

\section{Un ensemble de questions pour la didactique}

\subsection{Questionner l'unité et la diversité}

\subsubsection{Conceptions}

Pour les plus jeunes enfants, le vivant est d'abord perçu dans sa diversité, dans la richesse des formes vivantes, animales et végétales. Comment susciter un questionnement sur l'unité du vivant? Des propositions d'activités sont faites (par exemple l'alternative "vivant ou non vivant" dans l'atelier vécu à Montpellier). Des recherches seraient nécessaires, cependant, pour mieux analyser :

- les conceptions des élèves,

- les obstacles de conceptualisation dans l'articulation de l'unité et de la diversité du vivant.

\subsubsection{Niveaux de formulation}

Différentes recherches sur les niveaux de formulation, à l'école élémentaire, de l'unité et de la diversité du vivant ont été réalisées (INRP 1976). Ne faudrait-il pas reprendre la problématique de la conceptualisation dans différents cadres de références à tous les niveaux d'enseignement? 


\subsubsection{Idéologies implicites. Obstacles}

Unité et diversité ne sont pas des dénominations neutres. Elles peuvent véhiculer, implicitement, des valeurs et des idéologies, en particulier politiques, socialistes ou libérales. Dans le dictionnaire (Petit Robert 1994), l'unité est définie comme la régularité, l'uniformité, la conformité mais aussi comme la communauté, la cohésion, l'homogénéité et l'harmonie ; la diversité, de son côté, représente « ce qui est changeant » mais aussi le dissemblable, la multiplicité, la pluralité et l'hétérogénéité. L'unité est parfois influencée par des idéologies «totalitaires » et normatives »; la recherche de lois unificatrices, l'élégance de la simplicité mais au détriment de l'originalité, de la relativité et de la compréhension réelle de la complexité.

\begin{tabular}{|l|l|}
\hline \multicolumn{1}{|c|}{ UNITÉ } & \multicolumn{1}{|c|}{ DIVERSITÉ } \\
\hline Communauté & $\begin{array}{l}\text { Unicité } \\
\text { Singularité } \\
\text { Particularité }\end{array}$ \\
\hline Stabilité & Variabilité \\
\hline Simplicité & Complexité \\
\hline Ordre & Désordre \\
\hline Réductionnisme & $\begin{array}{l}\text { Holisme } \\
\text { Évolutionnisme } \\
\text { Écologisme }\end{array}$ \\
\hline
\end{tabular}

Il y a aussi des risques de confusion. Toujours dans le dictionnaire, l'unité est définie comme «ce qui est unique »: un qualificatif qui, en biologie, caractérise justement la diversité des êtres vivants. Il convient donc de rester vigilant, de prendre garde aussi de distinguer l'unité de la totalité (l'unité de l'organisme), l'unité de la continuité.

La question des démarches pédagogiques à mettre en place se pose également. Comment conjuguer les logiques apparemment contradictoires de l'aspect universel d'une « loi » et de la variabilité des réponses (intra-individuelles, individuelles et spécifiques) ? Faudraitil mieux envisager une juxtaposition des concepts d'unité et de diversité, ou bien envisager une approche plus dialectique ou plus dialogique ? Peut-on articuler à la fois une unité à briser (avec la vigilance, les exceptions, les limites de validité, les variations,...) et une diversité à rassembler ?

\subsection{Regarder vers d'autres sciences}

Un questionnement croisé entre sciences peut se révéler fécond. Ainsi l'unité, avec la recherche de lois unificatrices, constitue un paradigme essentiel pour les physiciens, tandis que les technologues, confrontés au problème de la variabilité (par exemple dans les constructions en série), doivent appréhender la variabilité avec la question de la 
norme et des erreurs. La pensée biologiste, d'autre part, s'enrichirait d'une confrontation avec des sciences plus modélisées et aléatoires, telle la climatologie.

\begin{tabular}{|c|c|}
\hline $\begin{array}{c}\text { Questions à } \\
\text { la biologie et à d'autres sciences }\end{array}$ & $\begin{array}{l}\text { Questions à } \\
\text { la didactique de la biologie }\end{array}$ \\
\hline Questions liées à l'unité (physique)? & Différents regards? \\
\hline Questions liées à la diversité (technologie)? & $\begin{array}{l}\text { Dialectique ou dialogue? } \\
\text { Equilibre des approches? }\end{array}$ \\
\hline $\begin{array}{l}\text { Unité du vivant et diversité des } \\
\text { formes vivantes: } \\
\text { - Signification du concept aux différents } \\
\text { niveau d'organisation du vivant? } \\
\text { - Questions de recherches actuelles? }\end{array}$ & $\begin{array}{l}\text { Conceptions? } \\
\text { Significations dans la vie quotidienne? } \\
\text { Choix des concepts à enseigner } \\
\text { Transposition didactique? } \\
\text { Niveaux de formulation? } \\
\text { Trames conceptuelles }\end{array}$ \\
\hline $\begin{array}{l}\text { Biologie: science plus modélisée et plus } \\
\text { probabiliste (exemple de la climatologie)? }\end{array}$ & $\begin{array}{l}\text { Quelle image de la biologie est } \\
\text { développée dans l'enseignement? }\end{array}$ \\
\hline
\end{tabular}

\section{Conclusion}

Penser à la fois l'unité et la diversité et reconnaitre un statut de science plus aléatoire semblent deux enjeux majeurs de la compréhension en biologie. Un rapprochement avec d'autres sciences qui rencontrent des difficultés analogues pourrait se révéler très fructueux. La technologie se trouve, par exemple, confrontée à des problèmes de compréhension de la variation. La climatologie, de son côté, doit gérer à la fois la complexité des interactions entre phénomènes et l'aléatoire.

Au-delà des problèmes pratiques concernant la formation des enseignants et la conciliation d'une approche naturaliste au collège avec l'effondrement des enseignements de sciences naturelles dans les Universités, il existe bien d'autres obstacles à l'intégration dans l'enseignement de «l'unité du vivant et de la diversité des formes vivantes ». Doit-elle prendre une forme équilibrée, dialectique ou dialogique ? Les questions ne manquent pas, à nous de nous atteler à la tâche et à la réflexion didactique !

\section{BIBLIOGRAPHIE}

ATLAN H., Entre le cristal et la fumée, Paris, Seuil, 1979.

BERNARD C., Introduction à l'étude de la médecine expérimentale, Paris, Flammarion, 1865, Réedition 1984.

CANGUILHEM G., La connaissante de la vie. Paris,Vrin, 1965, 2éd. 1980.

CANGUILHEM G., Le normal et le pathologique, Paris, PUF, 1966.

CANGUILHEM G., Études d'histoire et de philosophie des sciences, Paris, Vrin, 1968, 5e éd. 1983.

CANGUILHEM G., Idéologie et rationalité dans l'histoire des sciences de la vie, Paris, Vrin, $2^{\mathrm{e}}$ éd., 1981. 
CLEMENT P., « De la régulation à l'auto-organisation », in La régulation en biologie, Approche didactique : représentation, conceptualisation, modélisation, coord. : RUMELHARD G., Paris, INRP,Collection : Didactiques des Disciplines, 1995, p. 7-24.

COLL., Activités d'éveil scientifiques, 4, Initiation biologique, Paris, INRP, 1976.

DAGOGNET F., Le vivant, Paris, Bordas. 1988.

DROUIN J.-M., « L'image des sociétés d'insectes en France à l'époque de la Révolution », in Revue de synthèse "Animalité et anthropomorphisme dans la diffusion et la vulgarisation scientifiques ", № 3-4, Paris, Albin Michel, 1992, p. 333-346.

FOUCAULT M., Les mots et les choses, Paris, Gallimard, 1966.

GAYON J. « Animalité et végétalité dans les représentations de l'hérédité », in Revue de Synthèse "Animalité et anthropomorphisme dans la diffusion et la vulgarisation scientifiques ", No 3-4, Paris, Albin Michel, 1992, p. 423-438.

GAYON J., « La Biologie entre loi et histoire », in Philosophie, N 38, Paris, Éd. de Minuit, 1993, p. $30-57$.

GOULD S. J., Un hérisson dans la tempête, Traduction 1994, Paris, Grasset, 1987.

GOULD S. J., "Ladders and Cones : Constraining Evolution by Canonical Icons", in New York Review, éd. : SILBERSB., New York, USA, Collection : Hidden Histories of Science, 1995, p. 37-68.

JACOB F., La logique du vivant. Une histoire de l'hérédité, Paris, Gallimard, 1970.

KOURILSKY P., «Des herbiers aux génomes », in intervention Journées d'études sur la culture scientifique des professeurs des Écoles, Paris, La Villette, Cité des Sciences et de l'Industrie, janvier 1993.

LUCAS A., "Jouer les notes sans connaître la mélodie : le caractère étroit de la recherche en didactique de la biologie », in Disdaskalia, $N^{\circ}$ 1, Paris, INRP \& Université Laval (Québec, Canada), 1993, p. 101-113.

MAYR E., « Le chaos en biologie », in La Recherche, $\mathrm{N}^{\circ} 232$, 1991. p. 588-598.

MAYR E., Histoire de la biologie. Diversité, évolution et hérédité, Traduction 1989, Paris, Fayard, 1982. McCLINTOCK B., “A cytological and genetical study of triploid maize”, in Genetics. N 14, 1929, p. 180-222.

MCCLINTOCK B., "Chromosome organization and genic expression”, in Actes de Colloque : Cold Spring Harbor Symposia Quant. Biol., N 16, 1951, p. 13-47.

PICHOT A., Histoire de la notion de vie, Paris, Gallimard, 1993.

ROSTAND J., Carnets d'un Biologiste, Paris, Stock, 1959.

RUMELHARD G., « De la biologie contemporaine à son enseignement », in Savoirs scolaires et didactiques des disciplines : une encyclopédie pour aujourd'hui, dir. : DeVELAY M., Paris, ESF, 1995.

SCHIFF M., L'homme occulté. Le citoyen face au scientifique. Paris, Les Editions Ouvrières, Collection : «Portes ouvertes », 1992.

SCHWARTZ D., Le jeu de la science et du hasard. La statistique et le vivant, Paris, Flammarion, 1994.

COURRIER INTERNATIONAL, Nº 237 (18/5/95) et No 253 (7/9/95).

MANUELS SCOLAIRES, $2^{\text {de }}, 1^{\text {re }}$ et Term S, Éditions Bordas, Hachette et Nathan, 1994. 


\section{RÉSUMÉS}

L'unité et la diversité du vivant à tous les niveaux d'intégration est un concept fondamental de la biologie moderne. Suivant les périodes et selon les différents domaines d'études biologiques, la priorité a souvent été donnée à l'un ou l'autre aspect de ce « concept bicéphale ». Penser à la fois l'unité et la diversité semble un enjeu majeur de la compréhension en biologie. La comparaison avec d'autres disciplines scientifiques soulève de nombreuses questions didactiques.

The oneness and diversity common to all life forms is a fundamental concept of modern biology. Depending upon the historical period and the various fields in the study of biology, priority bas often been given to one or the other aspect of this two-sided concept. Thinking through the seeming paradox of oneness and diversity appears to be a major stumbling block to comprehension in biology. Comparison with other scientic disciplines raises a number of didactic issues.

\section{INDEX}

Mots-clés : diversité, histoire, question didactique, unité, vivant

Keywords : didactic issue, diversity, history, living thing, oneness

\section{AUTEUR}

\section{MARYLINE CANTOR}

IUFM de Haute-Normandie et université de Rouen, laboratoire LIREST/GDSTC, ENS Cachan 